\title{
Inter-related in vitro effects of androgens, fatty acids and oxidative stress in prostate cancer: A mechanistic model supporting prevention strategies
}

\author{
HELEN LIN $^{1}$, JIAN-PING LU ${ }^{1,2}$, PIERRE LAFLAMME ${ }^{1}$, SHENGJUN QIAO ${ }^{1,2}$, \\ BOBBY SHAYEGAN ${ }^{2}$, INNA BRYSKIN ${ }^{4}$, LAUREN MONARDO ${ }^{1}$, BRIAN C. WILSON ${ }^{4}$, \\ GURMIT SINGH $^{1,3}$ and JEHONATHAN H. PINTHUS ${ }^{1,2}$
}

\author{
${ }^{1}$ Juravinski Cancer Centre, Departments of ${ }^{2}$ Surgery, ${ }^{3}$ Pathology and Molecular Medicine, McMaster University, Hamilton \\ Ontario; ${ }^{4}$ Department of Medical Biophysics, Ontario Cancer Institute/University of Toronto, Toronto, Ontario, Canada
}

Received May 6, 2010; Accepted June 17, 2010

DOI: 10.3892/ijo_00000725

\begin{abstract}
Oxidation of mitochondrial fatty acids (FA) results in the generation of reactive oxygen species (ROS) which have been postulated to play a key role in the initiation and progression of prostate cancer (PC). We previously reported that androgens increase FA uptake into PC cells. We thus examined if androgens that are known to induce ROS generation regulate FA oxidation in PC cells. The effects of the androgen-depleted medium, R1881 (synthetic androgen) and/ or androgen receptor blocker, bicalutamide were examined in the human androgen-responsive but not dependent 22rv1 cells. R1881 supplementation significantly increased mitochondrial FA oxidation $\left({ }^{14} \mathrm{C}\right.$-radiolabeled FA degradation studies), resulting in increased ROS production. Androgens increased the mRNA levels of carnitine palmitoyltransferase (CPT1), the rate limiting enzyme in the process of mitochondrial FA oxidation. Treatment with R1881 and bicalutamide inhibited these androgen regulated effects. Inhibition of mitochondrial ROS generation by two different inhibitors, rotenone and thenoyltrifluoroacetone, eliminated the androgeninduced ROS generation, to the same level as in cells deprived of androgens or treated with R1881 and bicalutamide. Taken together, androgens increase the mitochondrial oxidation of FA, leading to increased production of ROS that is associated with prostate cell proliferation and mutagenesis. These results therefore support the rationale for PC prevention using 5- $\alpha$ reductase inhibitors, dietary restrictions or anti-oxidants, each of which has different inhibitory but complementary effects.
\end{abstract}

Correspondence to: Dr Jehonathan H. Pinthus, Department of Surgical Oncology, Juravinski Cancer Centre, 699 Concession Street, Hamilton, Ontario L8V 5C2, Canada

E-mail: jehonathan.pinthus@jcc.hhsc.ca

Key words: androgens, fatty acids, oxidative stress, mitochondria, prostate cancer prevention

\section{Introduction}

Prostate cancer (PC) is currently the most common noncutaneous malignancy and the second leading cause of cancer death in American men (1). PC is an attractive target for prevention strategies because it has a high incidence, a long protracted latent course and defined risk factors. Descriptive epidemiologic data suggest that androgens, genetic susceptibility and environmental exposures, such as diet (in particular, dietary fat and low anti-oxidant compounds), play an important role in prostatic carcinogenesis (2). A possible link between all these risk factors is the generation of oxidative stress within prostatic cells $(2,3)$. Oxidative stress is a key factor in the initiation and development of PC. It is caused by an imbalance between the production of reactive oxygen species (ROS) and their detoxification, triggering a host of pro-carcinogenic processes, and in particular accumulation of oxidative DNA damage. ROS have also been shown to exert regulatory roles in controlling cell proliferation, survival and differentiation. PC cells with elevated levels of ROS demonstrate enhanced cell signaling and activation of target genes that promote their survival and progression $(4,5)$. We (6) and others (7) have shown that androgens increase the production of ROS in PC cells, but the mechanism(s) remain unclear.

Recently, we also demonstrated that androgens increase the uptake of medium- and long-chain fatty acids into PC cells (8). These fatty acids can then either serve as building blocks for triacylglycerols and major membrane lipids, as has been extensively shown for PC (9), and/or potentially be directed to degradation. Surprisingly, there is limited data on fatty acid degradation (oxidation) and its regulatory mechanisms in PC. This is a pivotal issue, since a significant proportion of cellular ROS production is coupled to mitochondrial fatty acid oxidation. Since increased fatty acid content in the diet is a known risk factor for the development of PC, potentially through the induction of oxidative stress, we hypothesize that androgens regulate mitochondrial fatty acid degradation. This links three different risk factors for PC: androgen stimulation, fatty acids and oxidative stress. 


\section{Materials and methods}

In vitro experimental models. The androgen-responsive 22Rv1 human PC cell line was purchased from the American Type Culture Collection (Manassas, VA). Cells were grown in androgen-depleted medium composed of phenol-free RPMI-1640 medium, 10\% charcoal-stripped fetal bovine serum (csFBS) (Gibco, Grand Island, NY), 10 mM Hepes (Gibco), $1.0 \mathrm{mM}$ sodium pyruvate (Gibco ), $2 \mathrm{mM}$ L-glutamine (Invitrogen) and $1.0 \%$ penicillin and streptomycin (Gibco). Cell lines were cultured at $37^{\circ} \mathrm{C}$ in a humidified atmosphere containing $5.0 \% \mathrm{CO}_{2}$.

Hormonal treatments. The synthetic androgen R1881 (Sigma-Aldrich, Oakville, ON, Canada) was added to a final concentration of $10 \mathrm{nM}$ to create an androgen-supplemented medium. This concentration was selected to recapitulate the normal values for testosterone in the serum of adult males (14-35 nM). To block the effects of testosterone, the androgen receptor blocker bicalutamide (Astra Zeneca, Macclesfield, $\mathrm{UK}$ ) was added to a final concentration of $10 \mu \mathrm{M}$, reproducing the mean plasma concentration $(50.2 \mu \mathrm{M})$ in PC patients treated with bicalutamide monotherapy (150 mg daily) (10). Cells were pre-incubated in serum-starved media containing $0.5 \%$ FBS for 24-72 $\mathrm{h}$ before further manipulation. Incubation with the different hormonal treatments was for 48-72 h.

Nitroblue tetrazolium (NBT) assay. Cells were grown to $80 \%$ confluence in 96-well plates. R1881 and/or bicalutamide were added $48 \mathrm{~h}$ before the experiment. Rotenone $(100 \mu \mathrm{M}$, Sigma-Aldrich), thenoyltrifluoroacetone (PTFA: $150 \mu \mathrm{M}$, Sigma-Aldrich) and N-acetyl cystein (NAC: $10 \mathrm{mM}$, SigmaAldrich) were added $1 \mathrm{~h}$ before the experiment. ROS production was detected by the NBT assay (Sigma-Aldrich) as previously described (6). Briefly, cells were incubated for $90 \mathrm{~min}$ in phosphate-buffered saline (PBS) containing $0.1 \%$ NBT. The formazan crystals that were generated from the reduction of the NBT by the ROS were dissolved by sonication in $50 \%$ acetic acid, and the optical absorbance was determined at $560 \mathrm{~nm}$. After the subtraction of assay blanks, the results were normalized to the metabolic activity of cells in each hormonal condition, as determined by the WST-1 assay (Roche, Quebec, Canada). Briefly, the tetrazolium salt WST-1 is converted into a colored dye by mitochondrial dehydrogenase enzymes. The soluble salt is released into the media producing a color change (read at $450 \mathrm{~nm}$ with a reference reading at $630 \mathrm{~nm}$ ) which is directly proportional to the amount of mitochondrial dehydrogenase, a reflection of the net metabolic activity of the cells.

Confocal microscopy. Cells were cultured for $72 \mathrm{~h}$ under the different hormonal conditions described above. One hour before the experiments, $100 \mu \mathrm{M}$ Rotenone, $150 \mu \mathrm{M}$ PTFA or $10 \mathrm{mM}$ NAC was added to the medium. Cells were harvested and placed onto glass cover slips at a density of $10^{3}$ cells $/ \mathrm{ml}$. Cells were stained with $5 \mu \mathrm{M}$ dihydroethidium (DHE, Molecular Probes, Burlington, Ontario, Canada). After $24 \mathrm{~h}$, the cells were loaded with $5 \mu \mathrm{M}$ dihydroethidium (DHE, Molecular Probes) for $30 \mathrm{~min}$ at $37^{\circ} \mathrm{C}$. After two PBS washes, fresh medium was added. DHE is oxidized by ROS to yield ethidium, the fluorescence of which was measured with a confocal fluorescence microscope (excitation $=475 \mathrm{~nm}$, emission $=610 \mathrm{~nm}$ ). Images were analyzed with Image Pro software (Media Cybernetics, Silver Spring, MD).

Fatty acid degradation assay. The 22Rv1 cells were grown to sub-confluence and treated with the different hormonal conditions described above in RPMI-1640 medium without phenol red plus $0.5 \%$ csFBS for $72 \mathrm{~h}$. Prior to adding ${ }^{14} \mathrm{C}$ palmitic acid (specific activity $793 \mathrm{mCi} / \mathrm{mmol}$ ) (GE Healthcare, QC, Canada) to the cells, $1 \mathrm{~cm}$ diameter Whatman fiber disks (GE Healthcare) pre-treated with $100 \mu 1$ of tissue solubilizer base (GE Healthcare) for $30 \mathrm{~min}$ were suspended by a pin from the underside of a rubber septum (Sigma-Aldrich). The cells were washed with $1 \mathrm{X}$ PBS twice, trypsinized and collected. After re-suspension in Dulbecco's phosphate buffered saline (DPBS), $2 \times 10^{6}$ cells in a volume of $1.5 \mathrm{ml}$ were added into each vial. Nine microliters of $0.20 \mu \mathrm{Ci} / \mu \mathrm{l}{ }^{14} \mathrm{C}$ palmitic acid were added to $351 \mu 1$ of dimethyl sulphoxide (DMSO) and mixed, and then $20 \mu 1$ was added to each vial (to receive $\sim 1.3 \% \mathrm{v} / \mathrm{v}$ DMSO) and swirled. The glass vials were then fitted with the rubber stopper-fiber disk to collect the ${ }^{14} \mathrm{C}$ labelled $\mathrm{CO}_{2}$ released from the oxidation of the ${ }^{14} \mathrm{C}$-palmitic acid, while avoiding contact between the disks and the cellbuffer mixture. The vials were then incubated at $37^{\circ} \mathrm{C}$ for $1 \mathrm{~h}$, with continuous gentle agitation throughout the incubation time. The disks were removed after $1 \mathrm{~h}$ and placed in scintillation plastic vials (Fisher, $\mathrm{AB}$ ) containing $5 \mathrm{ml}$ of scintillation cocktail (Ready Safe: Beckman, CA) and $50 \mu 1$ of glacial acetic acid (Caledon, ON, Canada). The counts were collected using a liquid scintillator system to measure $\beta$ emissions and expressed as disintegration per minute (DPM). Background counts from control vials containing PBS and ${ }^{14} \mathrm{C}$ palmitic acid were subtracted to obtain the final values.

Real-time quantitative PCR. Different hormonal conditions described above were applied to 22Rv1 cells for 24-48 h. Total RNA was extracted from the cells (Total RNA isolation kit, Molecular Bio, Carlsbad, CA) and treated with DNase (Sigma-Aldrich). The total RNA levels were normalized by UV spectrophotometry (Beckman DU 640B) and also by quantification of the 18S, 28S RNA transcript with Bio-Rad Quantity One software. Complement DNA (cDNA) was prepared from $5 \mu \mathrm{g}$ total RNA with Superscript III reverse transcriptase (Invitrogen, Carlsbad, CA). Relative quantitative real-time PCR of the CPTI liver isoenzyme (L-CPTI) and muscle isoenzyme (M-CPTI) was performed using following primers: L-CPTI forward 5'-GTCCCGGCTGTCAAAG ACA-3', reverse 5'-CCGACAGCAAAATCTTGAGCA-3' (amplified fragment size 109 bps) (11); M-CPTI forward 5'-CCGGGACAGGGGTAAGTTCT-3', reverse 5'-CTCCAT CATGGCCTGCACAA-3' (126 bps). PCR reaction was carried out in MiniOpticon System (Bio-Rad, Hercules, CA) with SYBR Green Supermix (Bio-Rad). The PCR conditions were: denaturation $94^{\circ} \mathrm{C} 3 \mathrm{~min}$; annealing $62^{\circ} \mathrm{C} 25 \mathrm{sec}$; extension $72^{\circ} \mathrm{C} 25 \mathrm{sec} ; 35$ cycles. All experiments were completed in triplicate with 4 repetitions using RNA isolated from a new batch of cells. The relative amount of target cDNA (reflecting mRNA level) was normalized to the amount of Bactin [(Primers: 5'-TCTACAATGAGCTGCGTGTG-3' 
a.

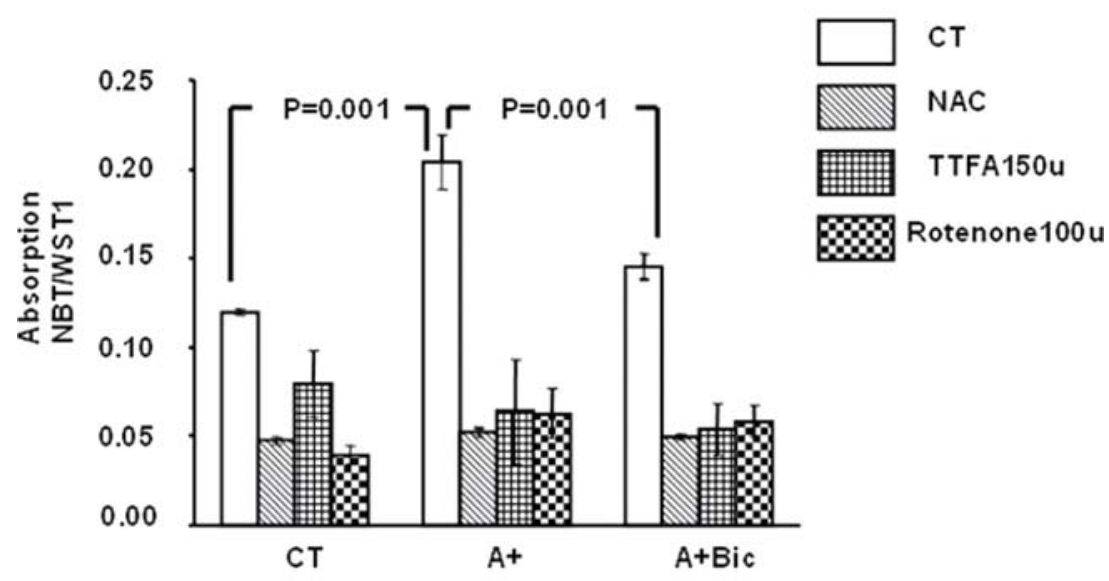

b.
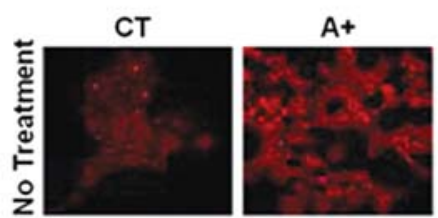

A+Bic
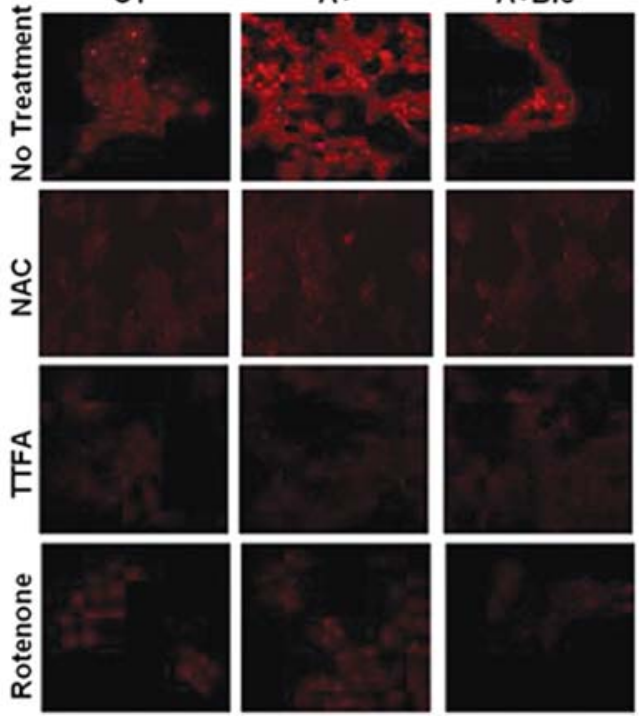

Figure1. Effect of androgens on mitochondrial production of ROS in 22rv1 cells. Cells were grown for $48 \mathrm{~h}$ in $96-$ well plates with or without R1881 and bicalutamide. Cells were than treated with $100 \mu \mathrm{M}$ Rotenone, $150 \mu \mathrm{M}$ thenoyltrifluoroacetone (PTFA) or $10 \mathrm{mM}$ N-acetyl cystein (NAC) for $1 \mathrm{~h}$ before conducting the NBT reduction assay (a) or the DHE confocal microscopy (b). Results are from one of at least three independent experiments (with 6 repetitions in each) with similar results. $\mathrm{CT}=$ androgen-depleted medium comprising phenol-free medium and $10 \% \mathrm{csFBS} ; \mathrm{A}+=\mathrm{Same}$ medium as $\mathrm{CT}+10 \mathrm{nM}$ $\mathrm{R} 1881 ; \mathrm{A}+\mathrm{Bic}=$ Same medium as CT $+10 \mathrm{nM} \mathrm{R} 1881$ and $10 \mu \mathrm{M}$ bicalutamide

(forward), 5'-AGCCTGGATAGCAACGTACA-3' (reverse) $(162 \mathrm{bps})]$. The mean mRNA levels of control cells were expressed as 1 to allow the comparison to the relative change of the mRNA levels under the experimental conditions (R1881 and R1881+ bicalutamide). All experiments were normalized using the $\Delta \Delta \mathrm{C}_{\mathrm{T}}$ method.

Statistical analysis. Statistically-significant differences between the 2 experimental groups were determined using the independent sample Student t-test with a 2-tailed value of $\mathrm{P}<0.05$ considered significant. Statistically-significant differences between the experimental groups and controls (of which the mean was achieved by averaging multiple repetitions is expressed as 100\% in Fig. 2 and as 1 in Fig. 3) were determined using one sample t-test with significance. Statistical analysis was performed using SPSS 12.0 (Chicago, IL).

\section{Results}

Androgens induce mitochondrial ROS production. To determine if the androgen induced increased production of ROS in PC cells originates from mitochondrial sources, we measured the induction of ROS under conditions of androgen supplementation and deprivation, with and without the addition of rotenone, an inhibitor of mitochondrial electron transport chain complex I, or thenoyltrifluoroacetone, an inhibitor of complex II, as well as the general ROS scavengerNAC (control). Cellular generation of ROS was examined using two independent methods: the NBT reduction assay (Fig. 1a) and DHE fluorescence imaging (Fig. 1b). As we previously described (6), incubation of 22rv1 cells with R1881 increased the production of ROS as compared to cells deprived of androgens $(p=0.001)$ or cells that were treated with both 


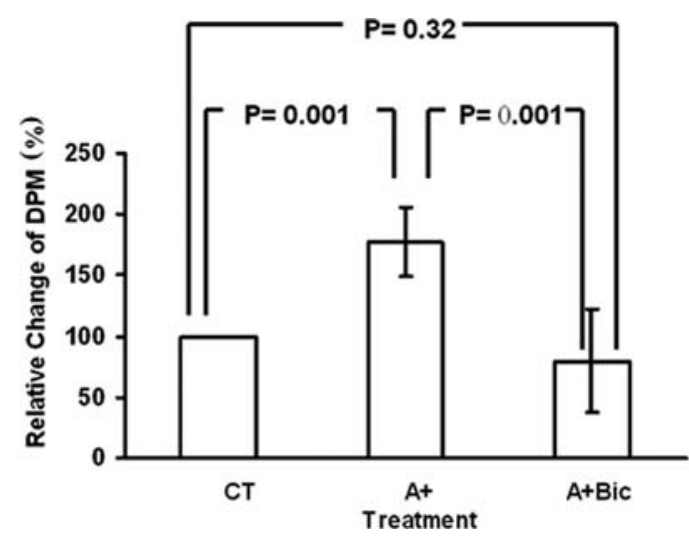

Figure 2. Effect of androgens on palmitic acid oxidation in 22rv1 cells. Results are from one of three independent experiments (each performed in triplicates) with similar results. Results are expressed as the change (\%) in DPM counts for $1 \mathrm{~h}{ }^{14} \mathrm{C}$-labelled $\mathrm{CO}_{2}$ released from the oxidation of the ${ }^{14} \mathrm{C}$ palmitic acid, compared to control. CT (control) = androgen-depleted medium comprising phenol-free medium and $10 \%$ csFBS; A+ = Same medium as CT + $10 \mathrm{nM}$ R1881; $\mathrm{A}+\mathrm{Bic}=$ Same medium as CT $+10 \mathrm{nM} \mathrm{R} 1881$ and $10 \mu \mathrm{M}$ bicalutamide

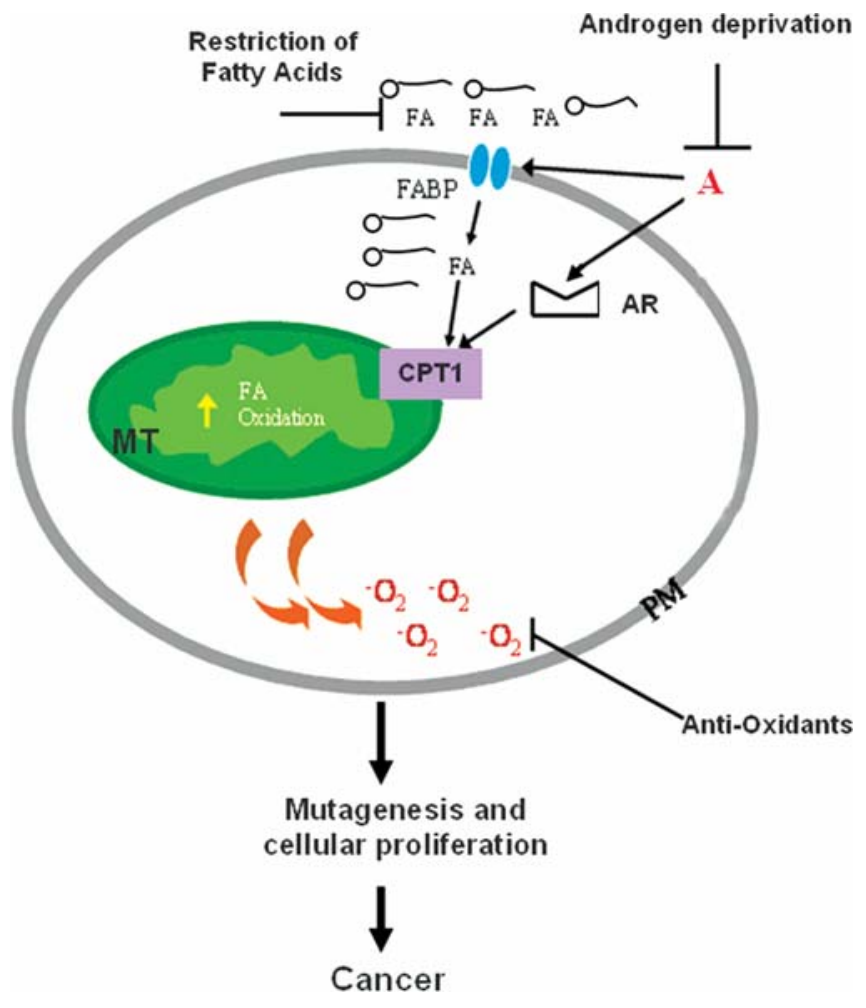

Figure 4. Integrated model of prostate cancer prevention strategies aiming to reduce oxidative stress. Androgens (A) through androgen-receptor activation (AR) increase the expression of fatty acid binding protein (FABP) on the plasma membrane (PM) of the cells, increasing the cellular uptake of fatty acids (FA) as shown previously (8). AR activation also increases the expression of carnitine palmitoyltransferase 1 (CPT1) to increase the mitochondrial (MT) uptake of FA leading to their oxidation and coupled generation of reactive oxygen species $\left(-\mathrm{O}_{2}\right)$. Cellular accumulation of reactive oxygen species induces mutagenesis and cellular proliferation leading to cancer. Reducing the availability of FA (dietary restriction), androgen deprivation or the use of anti-oxidants may have complementary inhibitory effects on different components in this model. a.

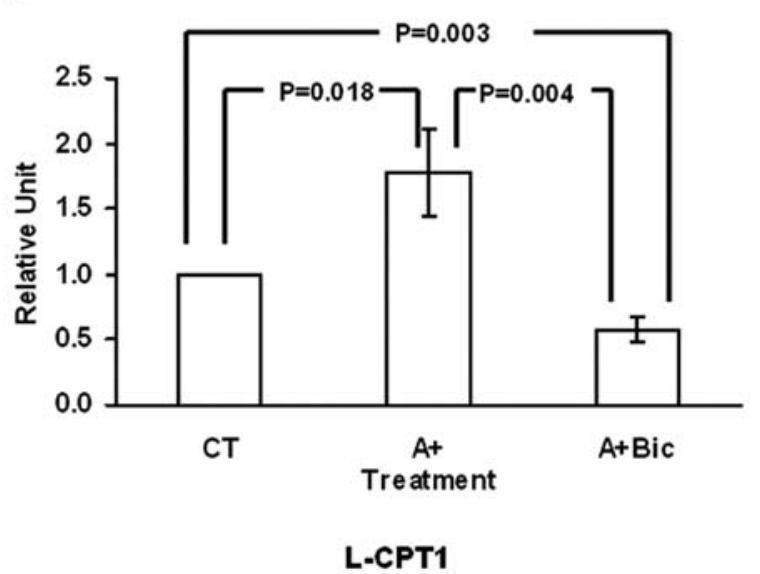

b.

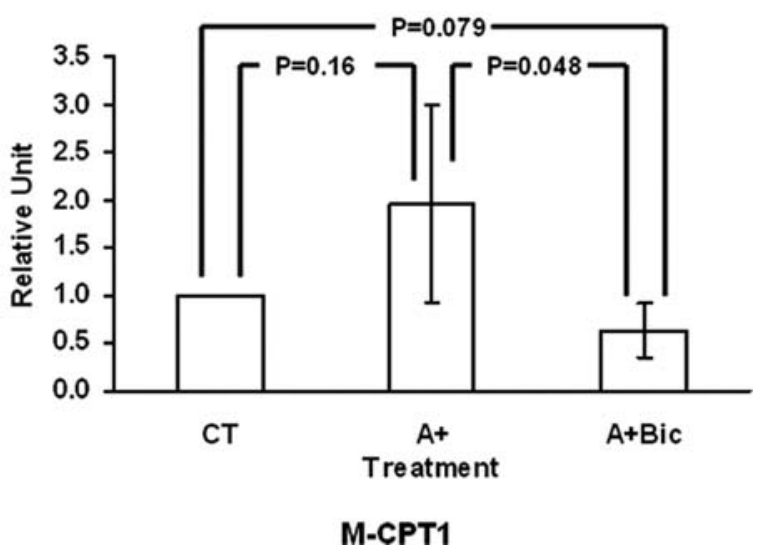

Figure 3. Effect of R1881 (A+) on the mRNA levels of L-CPTI (a) and MCPTI (b) isoenzymes compared to treatment with no androgens (CT) or with the combination of R1881 and bicalutamide (A+Bic) in 22rv1 cells. Results are expressed as the relative change in mRNA levels compared to control.

R1881 and bicalutamide ( $\mathrm{p}=0.001)$, indicating that androgens increase the mitochondrial production of ROS through androgen receptor mediated pathways (Fig. 1). However, the addition of rotenone or thenoyltrifluoroacetone significantly blocks the R1881 induced production of ROS equalizing the ROS levels across all hormonal conditions (Fig. 1). Collectively, these experiments confirm that androgens do indeed affect the mitochondrial production of ROS.

Androgens induce mitochondrial oxidation of fatty acids. Since androgens increase the mitochondrial production of ROS in PC cells, a process that is mainly coupled to mitochondrial $B$ oxidation of fatty acids (12), we examined if androgens regulate this biochemical pathway, by comparing the degradation of ${ }^{14} \mathrm{C}$-palmitic acid to radio labeled ${ }^{14} \mathrm{CO}_{2}$ under different hormonal conditions (Fig. 2). Treatment with R1881 significantly increased the degradation of ${ }^{14} \mathrm{C}$-palmitic acid compared to control cell treated with androgen deprivation ( $\mathrm{p}=0.001)$. Incubation with both $\mathrm{R} 1881$ and bicalutamide inhibited the degradation of ${ }^{14} \mathrm{C}$-palmitic acid to values similar to control androgen-deprived cells but significantly lower than in R1881-supplemented cells ( $\mathrm{p}=0.001$ ). 
Androgens positively regulate the transcription of carnitine palmitoyltransferase I (CPTI). In order to understand the mechanism by which androgens increase the mitochondrial oxidation of fatty acids, we investigated whether androgens regulate the expression of CPTI, the rate-limiting enzyme in the fatty acid oxidation pathway. CPTI is located on the outer mitochondrial membrane and catalyzes the transfer of a longchain fatty acyl group from coenzyme A to carnitine in order to shuttle fatty acids into the mitochondria. CPTI has 2 isoenzymes: liver form (L-CPTI) and muscle form (M-CPTI). To our knowledge, the expression of both isoenzymes has never been described in PC. In the absence of compatible antibodies to L-CPTI and M-CPTI that would allow testing of the protein levels, we used real-time quantitative PCR to examine the effects of androgen on the transcript level. We found that androgens significantly increased the mRNA expression of the L-CPTI isoenzyme in 22rv1 PC cells (Fig. 3a). Incubation with both androgens and the androgen receptor blocker bicalutamide reduced the mRNA expression of the L-CPTI isoenzyme to levels lower than that in control androgen deprived cells $(\mathrm{p}=0.003)$. Although androgens increased the mRNA expression of the M-CPTI isoenzyme over control androgen deprived cells (Fig. 3b) this did not reach statistical significance $(\mathrm{p}=0.16)$. However, incubation with both androgens and bicalutamide significantly inhibited the androgen induced expression of M-CPTI mRNA compared to androgen supplemented cells $(\mathrm{p}=0.048)$. These results suggest that the mRNA expression of both CPTI isoenzymes is regulated in 22rv1 PC cells in an androgen receptor-mediated pathway.

\section{Discussion}

Although still a matter of debate, several epidemiological and experimental studies have suggested that fatty acids have a role in the development and progression of androgensensitive-PC (13-15). We examined the link between fatty acid metabolism and the effects of androgens on the generation of oxidative stress, a key event in prostate carcinogenesis (3). Thus, in the present study we tested in vitro the hypothesis that androgens regulate the production of ROS by increasing the mitochondrial oxidation of fatty acids.

The involvement of androgens in fatty acid metabolism occurs at multiple levels, from cellular uptake to oxidation or their conversion in the process of lipid synthesis. We previously demonstrated that androgens increase the cellular uptake of medium and long chain fatty acids by inducing the expression of one of the fatty acid membrane binding proteins, FABPpm (8). Upon uptake, the fatty acids can either be directed to fat synthesis or can serve as a metabolic fuel through their oxidation. This may be an important bioenergetic pathway in $\mathrm{PC}$, which is not a highly glycolytic tumor. Unlike most other tumors (16) and in contrast to the androgen-independent phase of PC, glucose is probably not required as a metabolic source for the slow growth of androgen-dependent PC cells (17). This suggests that androgens may regulate the utilization of an alternative energy production pathway, likely via fatty acid oxidation (18). Accordingly, several well documented alterations in the expression and/or activity of enzymes involved in this pathway characterize PC: loss of stearoyl-CoA desaturase (SCD) (19), an enzyme involved in the synthesis of mono-saturated fatty acids, causes accumulation of palmitate. This is a negative regulator of ACC, which consequently reduces the level of malonyl-CoA and thereby increasing the activity of CPTI, which is the rate-limiting step in fatty acid oxidation (20). Zha and colleagues (21) demonstrated that there is upregulation of enzymes involved in peroxisomal branched-chain fatty acid oxidation to medium and short chain fatty acids, which then undergo final $ß$-oxidation in the mitochondria. In addition, one of the characteristic changes in PC is overexpression of $\alpha$-methylacyl-CoA racemase (AMACR): over $95 \%$ of prostate cancers, of all grades and stages and in both hormone refractory and sensitive cases, stain positively for AMACR as compared to $<4 \%$ of histologically normal prostate epithelium (22). AMACR plays a critical role in the oxidation of branched chain fatty acids which originate almost entirely from the diet. These fatty acids contain methyl groups on carbon 2 (relative to the carboxyl group) in R-stereo-chemistry, whereas the enzymes of the $B$ oxidation pathway can only metabolize substrates having the $S$ epimer $(23,24)$. Hence AMACR, which catalyzes the interconversion between $\mathrm{R}$ and $\mathrm{S}$ stereo-isomers, facilitates fatty acid oxidation. Overall, PC cells seem to express enzymatic machinery that promotes fatty acid oxidation. Nevertheless, current data regarding the potential regulation of these enzymes by androgens is lacking or negative for AMACR $(25,26)$.

It is well established, however, that androgens induce lipogenesis in PC cells. Androgens regulate the expression of multiple genes involved in lipid metabolism (9). In particular, androgens increase the gene expression and activity of fatty acid synthase (FAS), a key enzyme in fatty acid synthesis that catalyzes the synthesis of fatty acids from acetyl-CoA and Malony-CoA (9). In fact, increased FAS expression has been shown to correlate with poor prognosis and with the development of androgen-independent disease (27). Although it was clearly demonstrated that androgens increase the enzymatic activity of FAS, these studies were conducted following exogenous supplementation of ${ }^{14} \mathrm{C}$-labeled malonylCoA, the substrate for FAS (28). To our knowledge, no study has actually measured the levels of malonyl-CoA in PC cells following androgen supplementation or deprivation.

The fact that androgens stimulate lipogenesis does not contradict our findings. In fact, it is possible that androgens induce the activation of both lipogenesis and fatty acid degradation in a coordinated fashion. Accordingly, androgens stimulate the activity of FAS, leading to the accumulation of palmitate that in turn inhibits ACC, thereby shifting the pathway towards fatty acid oxidation. That is, through the induction of lipogenesis and accumulation of palmitate, androgens induce feedback inhibition on ACC, thus ultimately increasing the rate of fatty acid oxidation. This model explains the dual role of androgens: first, enhancing lipogenesis and second, subsequently facilitating fatty acid oxidation in androgen-dependent or androgen-responsive PC. Accordingly, with the pro-proliferative effects of androgens there is a demand for increased building blocks for membranes (phospholipids) and hence there is a rationale for increased lipogenesis, but this is accompanied by higher energy 
requirement that is addressed by subsequent enhancement of fatty acid oxidation.

Our findings also contribute to support the rational for PC prevention: given the central role of long-term exposure to androgens, high dietary fat content, and oxidative stress in the initiation and progression of $\mathrm{PC}$, our findings provide a potential integrated model to explain the combined contribution of all 3 risk factors to PC carcinogenesis (Fig. 4). The uptake of fatty acids into PC cells is androgen regulated through the increased expression of FABPpm (8). Therefore, more fatty acids are available for mitochondrial oxidation, a process that is also positively regulated by androgens, possibly due to increase expression of CPTI. Increased fatty acid oxidation leads to increased production of ROS that is associated with cancer cell proliferation and mutagenesis. These results therefore support the rationale for PC prevention using $5-\alpha$ reductase inhibitors $(29,30)$ dietary restrictions or antioxidants, each of which have different inhibitory but complementary effects.

This study was conducted using only a single PC cell line. The objective however was to use a prototype human PC cells as a model system to prove a concept. In this respect the 22rv1 cells, which express the androgen receptor and are androgen responsive and not dependent is an ideal model as it can be manipulated by androgens/androgen deprivation and maintain metabolic activity and proliferation under both conditions.

This study was conducted in vitro to delineate the potential effect of androgens on mitochondrial FA oxidation. In vivo studies are planned to investigate the effects of androgens on the enzymatic activity of CPT1, in addition to altering its expression as demonstrated here in vitro.

\section{Acknowledgements}

We gratefully acknowledge Astra Zeneca for supplying us with bicalutamide. Astra Zeneca played no role in the design of the study, in the interpretation of the data or in the writing of the report. This study was supported by the Canadian Institutes of Health Research (C.I.H.R.) grant (to G.S., J.H.P. and P.L.), a research grant from the Juravinski Cancer Centre Foundation (to J.H.P.) and a scholarship from the Canadian Urological Association (to J.H.P.).

\section{References}

1. Jemal A, Siegel R, Ward E, et al: Cancer statistics, 2006. CA Cancer J Clin 56: 106-130, 2006.

2. Fleshner NE and Klotz LH: Diet, androgens, oxidative stress and prostate cancer susceptibility. Cancer Metastasis Rev 17: 325-330, 1998

3. Klein EA, Casey G and Silverman R: Genetic susceptibility and oxidative stress in prostate cancer: Integrated model with implications for prevention. Urology 68: 1145-1151, 2006.

4. Sung SY, Kubo H, Shigemura K, et al: Oxidative stress induces ADAM9 protein expression in human prostate cancer cells. Cancer Res 66: 9519-9526, 2006.

5. Shigemura K, Sung SY, Kubo H, et al: Reactive oxygen species mediate androgen receptor- and serum starvation-elicited downstream signaling of ADAM9 expression in human prostate cancer cells. Prostate 67: 722-731, 2007.

6. Pinthus JH, Bryskin I, Trachtenberg J, et al: Androgen induces adaptation to oxidative stress in prostate cancer: Implications for treatment with radiation therapy. Neoplasia 9: 68-80, 2007.
7. Ripple MO, Henry WF, Rago RP, et al: Prooxidant-antioxidant shift induced by androgen treatment of human prostate carcinoma cells. J Natl Cancer Inst 89: 40-48, 1997.

8. Pinthus JH, Lu JP, Bidaisee LA, et al: Androgen-dependent regulation of medium and long chain fatty acids uptake in prostate cancer. Prostate 67: 1330-1338, 2007.

9. Swinnen JV, Esquenet M, Goossens K, Heyns W and Verhoeven G: Androgens stimulate fatty acid synthase in the human prostate cancer cell line LNCAP. Cancer Res 57: 10861090, 1997.

10. Tyrrell CJ, Denis L, Newling D, Soloway M, Channer K and Cockshott ID: Casodex 10-200 mg daily, used as monotherapy for the treatment of patients with advanced prostate cancer. An overview of the efficacy, tolerability and pharmacokinetics from three phase II dose-ranging studies. Casodex study group. Eur Urol 33: 39-53, 1998.

11. Razeghi P, Young ME, Alcorn JL, Moravec CS, Frazier OH and Taegtmeyer H: Metabolic gene expression in fetal and failing human heart. Circulation 104: 2923-2931, 2001.

12. Nubel T and Ricquier D: Respiration under control of uncoupling proteins: Clinical perspective. Horm Res 65: 300-310, 2006.

13. Clinton SK and Giovannucci E: Diet, nutrition, and prostate cancer. Annu Rev Nutr 18: 413-440, 1998.

14. Ramon JM, Bou R, Romea S, et al: Dietary fat intake and prostate cancer risk: A case-control study in Spain. Cancer Causes Control 11: 679-685, 2000.

15. Kolonel LN, Nomura AM and Cooney RV: Dietary fat and prostate cancer: Current status. J Natl Cancer Inst 91: 414-428, 1999.

16. Warburg O: On respiratory impairment in cancer cells. Science 124: 269-270, 1956.

17. Singh G, Lakkis CL, Laucirica R and Epner DE: Regulation of prostate cancer cell division by glucose. J Cell Physiol 180: 431-438, 1999.

18. Liu Y: Fatty acid oxidation is a dominant bioenergetic pathway in prostate cancer. Prostate Cancer Prostatic Dis 9: 230-234, 2006.

19. Moore S, Knudsen B, True LD, et al: Loss of stearoyl-CoA desaturase expression is a frequent event in prostate carcinoma. Int J Cancer 114: 563-571, 2005.

20. Ntambi JM, Miyazaki M, Stoehr JP, et al: Loss of stearoyl-CoA desaturase-1 function protects mice against adiposity. Proc Natl Acad Sci USA 99: 11482-11486, 2002.

21. Zha S, Ferdinandusse S, Hicks JL, et al: Peroxisomal branched chain fatty acid beta-oxidation pathway is upregulated in prostate cancer. Prostate 63: 316-323, 2005.

22. Luo J, Zha S, Gage WR, et al: Alpha-methylacyl-CoA racemase: A new molecular marker for prostate cancer. Cancer Res 62: 2220-2226, 2002.

23. Wanders RJ, Vreken P, Ferdinandusse S, et al: Peroxisomal fatty acid alpha- and beta-oxidation in humans: Enzymology, peroxisomal metabolite transporters and peroxisomal diseases. Biochem Soc Trans 29: 250-267, 2001.

24. Lloyd MD, Darley DJ, Wierzbicki AS and Threadgill MD: Alpha-methylacyl-CoA racemase-an 'obscure' metabolic enzyme takes centre stage. FEBS J 275: 1089-1102, 2008.

25. Chen W, Wu W, Zhao J, et al: Molecular cloning and preliminary analysis of the human alpha-methylacyl-CoA racemase promoter. Mol Biol Rep 36: 423-430, 2009.

26. Zha S, Ferdinandusse S, Denis S, et al: Alpha-methylacyl-CoA racemase as an androgen-independent growth modifier in prostate cancer. Cancer Res 63: 7365-7376, 2003.

27. Rossi S, Graner E, Febbo P, et al: Fatty acid synthase expression defines distinct molecular signatures in prostate cancer. Mol Cancer Res 1: 707-715, 2003.

28. Schmidt LJ, Ballman KV and Tindall DJ: Inhibition of fatty acid synthase activity in prostate cancer cells by dutasteride. Prostate 67: 1111-1120, 2007.

29. Thompson IM, Goodman PJ, Tangen CM, et al: The influence of finasteride on the development of prostate cancer. N Engl J Med 349: 215-224, 2003.

30. Andriole GL: Overview of pivotal studies for prostate cancer risk reduction, past and present. Urology 73: S36-S43, 2009. 